\title{
COMPARACIÓN DE MODELOS DE RADIOPROPAGACIÓN EN EL ÁREA SUBURBANA DE LA CIUDAD DE BARRANQUILLA
}

\section{COMPARISON OF RADIO PROPAGATION MODELS IN THE SUBURBAN AREA OF THE CITY OF BARRANQUILLA}

\author{
MSc. Alexis Barrios Ulloa*, MSc. Ricardo Arjona Angarita*, \\ PhD. Ramón Álvarez López** \\ * Universidad Tecnológica de Bolívar, Facultad de Ingeniería, Grupo GAICO. \\ Kilómetro 1 vía Turbaco, Cartagena, Bolívar, Colombia. \\ (57)3015015149 \\ E-mail: alexisbarriosu@ hotmail.com,rarjona@unitecnologica.edu.co \\ ${ }^{* *}$ Universidad de Sucre, Departamento de Ingeniería Electrónica. \\ Sede Puerta Roja, Sincelejo, Sucre, Colombia. \\ (057) 52771195 \\ E-mail: ramon.alvarez@unisucre.edu.co
}

Resumen: En este artículo se evalúan tres modelos de propagación: COST-231 HATA, Walfisch Ikegami e Interino de la Universidad de Stanford para un escenario suburbano. Los datos fueron recolectados en un drive test en la banda de $1900 \mathrm{MHz}$, para lo cual se eligió un sector de la ciudad de Barranquilla, Colombia. Como método de evaluación, se utilizó el cálculo del error de predicción y relativo de cada modelo con respecto a las mediciones.

Los resultados obtenidos muestran que el modelo Interino de la Universidad de Stanford presentó niveles de error más bajos en relación a los obtenidos con el modelo COST-231 HATA y el Walfisch Ikegami.

Los altos valores de error permiten concluir que los modelos analizados no representan las mediciones en el escenario estudiado, lo cual hace necesario procesos de ajuste a fin de mejorar los resultados, además de ampliar la campaña de medición para validar los hallazgos.

Palabras clave Error de predicción, error relativo, escenario suburbano, modelos de propagación, pérdidas por trayectoria.

\begin{abstract}
This article describes three propagation models are evaluated: COST-231 Hata, Walfisch Ikegami Stanford University Interim for a suburban scenario. The Data were collected in a test drive at $1900 \mathrm{MHz}$ band, for which a sector of the city of Barranquilla, Colombia was elected. As a method of assessment, prediction error calculation and relative of each model with respect to the measurements we were used.

The results show that the model Interim Stanford University presented error levels lower relative to those obtained with the COST-231 model HATA and Walfisch Ikegami.

Higher error values can be concluded that the models do not represent the measurements analyzed in the study environment, which makes it necessary adjustment processes to improve the results, and expand the measurement campaign to validate the findings.
\end{abstract}

Keywords: Path loss, prediction error, Propagation models, relative error, suburban scenario.

\section{INTRODUCCIÓN}

El canal de propagación de radio es muy importante y un componente crítico para los sistemas de comunicación de radio móviles. Por lo tanto, su análisis detallado resulta importante para el diseño de futuros sistemas de comunicaciones 
móviles, así como para la optimización y extensión de sistemas existentes (Directorate-General Information society: Telecommunications, Markets, 1999).

Para diseñar y planificar una red inalámbrica es importante verificar los elementos que afectan los niveles de potencia de la señal en la línea de vista entre el transmisor y el receptor, debido a que existe una gran variabilidad de la señal por los efectos de los fenómenos físicos y aleatorios que dependen del ambiente y la región donde se desea implementar (Herrera y Pérez, 2008). Las paredes, muebles, vehículos y personas pueden causar la pérdida significativa de la señal. Las pérdidas de trayectoria cambian dramáticamente con el tiempo o la posición, debido a la cantidad de múltiples trayectos presentes y el movimiento de personas y elementos del entorno (Herrera y Hernández, 2016).

Los modelos de radiopropagación se encargan de determinar las pérdidas por trayectoria en un canal inalámbrico y son una medida de la atenuación de la señal y se pueden definir como la diferencia (en $\mathrm{dB}$ ) entre la potencia efectiva transmitida y la potencia recibida (Rappaport, 2002), y pueden ser clasificados en empíricos, determinísticos y estocásticos. Mientras que los empíricos se basan en mediciones, los determinísticos determinan las pérdidas por trayectoria a partir de los fenómenos de propagación y en un conocimiento adecuado del entorno. Por otro lado, los modelos estocásticos utilizan variables aleatorias para modelar el ambiente (Bhuvaneshwari y Sathyasavithri, 2013).

A pesar de sus limitaciones, los modelos empíricos presentan un buen desempeño en cuanto a precisión se refiere, sin embargo al ser concebidos para escenarios con características y mediciones específicas, pueden presentar altos valores de error cuando son usados en entornos totalmente distintos para los cuales fueron desarrollados originalmente; por lo cual es necesario probar la eficiencia de estos modelos en cada región o ambiente donde se desee aplicar, tal como ha sido estudiado en (Bhuvaneshwari y Sathyasavithri, 2013), (Ardila, 2012), (Herrera, 2013) y (Garcia y Rogriguez, 2002).

Barranquilla es uno de los centros urbanos más importantes de Colombia, y a lo largo de su geografía presenta diferentes tipos de escenarios urbanísticos de acuerdo a lo catalogado por la UIT en (ITU-R, 2013). La ciudad fue seleccionada para llevar a cabo un estudio comparativo de tres modelos de propagación: COST 231-HATA, Walfisch Ikegami e Interino de la Universidad de Stanford (SUI). En virtud de lo expuesto, el objetivo de este artículo es evaluar tres modelos de radiopropagación en un escenario urbanístico irregular del área suburbana de la ciudad de Barranquilla.

El resto del artículo está organizado de la siguiente forma: La sección II presenta una descripción de los modelos de propagación usados para este estudio. La sección III muestra el proceso de recolección de datos, y en la sección IV se presentan los resultados. Finalmente la sección V presenta las conclusiones.

\section{MODELOS DE PROPAGACIÓN}

\subsection{Modelo Cost-231}

El modelo COST 231-HATA fue planteado en 1993 y básicamente es una extensión a una banda de frecuencia más alta, $1500 \mathrm{MHz}$ a $2000 \mathrm{MHz}$, del modelo propuesto por Hata en 1980, válido para un rango de $150 \mathrm{MHz}$ a $1500 \mathrm{MHZ}$. Este último modelo es por sí mismo una formulación en ecuaciones de uno anterior desarrollado por Okumura y que en el momento de la ingeniería se hizo sobre la base de gráficos, tablas y reglas deslizantes. Este modelo se diseñó básicamente para ser utilizado en entornos de macrocélulas, es decir, para distancias entre la estación base (BS) y el terminal móvil (MT) entre 1 y 20 kilómetros por lo menos y es válido para alturas de la antena del transmisor entre 30 y 200 metros y para altura de la antena de la estación móvil entre 1 y 10 metros (Tahat y Alqudah, 2011). Las pérdidas por trayecto en este modelo están dadas por la ecuación (1):

$$
\begin{aligned}
& P L(d B)=46,3+33,9 \log _{10} f-13,82 \log _{10} h_{b}-a h_{m} \\
& +\left(44,9-6,55 \log _{10} h_{b}\right) \log _{10} d+C_{m}
\end{aligned}
$$

Donde $f$ es la frecuencia en Megahertz (MHz), $d$ es la distancia entre la estación base y el móvil en Kilómetros, $h_{b}$ es la altura de la antena de la estación base en metros. El parámetro $a h_{m}$ es un factor de corrección debido a la antena receptora que depende de la densidad estructural del entorno y para el ambiente suburbano viene dado por la ecuación (2):

$$
a h_{m}=3,2\left(\log _{10}\left(11.75 h_{r}\right)\right)^{2}-4,97
$$


Donde $h_{r}$ es la altura de la antena de la estación móvil en metros. El parámetro $C_{m}$ toma valor de 0 $\mathrm{dB}$ para ambientes suburbanos.

Una de las principales ventajas de este modelo es que permite mejor aproximación a las pérdidas por trayecto debido a la existencia de más datos que permiten describir el ambiente en el cual se aplica, en comparación a lo que ocurre con otros modelos como por ejemplo el de Espacio Libre. En este modelo, la dispersión Doppler, la propagación por trayectos y los efectos de desvanecimiento son tenidos en cuenta (Acar, 2015). Como desventajas se tienen una aproximación menor de las pérdidas cuando es usado en escenarios de microcélulas y que el modelo no considera las ondulaciones del terreno.

\subsection{Modelo Walfisch-Ikegami}

El modelo COST-231 - Walfisch- Ikegami fue desarrollado en 1993 y está basado en el modelo Walfisch-Bertoni. A pesar uno de los más complejos, es uno de los más usados en la actualidad. Es válido para alturas de la antena del transmisor ubicadas entre 4 y 50 metros de altura, para antenas de la estación móvil ubicadas entre 1 y 3 metros, distancias en un rango entre 200 a 5000 metros y para un rango de frecuencia que va de 800 a $2000 \mathrm{MHz}$. La figura 1 muestra los parámetros que definen el modelo de acuerdo a la recomendación UIT-R P.1411, el cual contempla situaciones sin línea directa (NLOS) y de línea directa (LOS) (Salazar y Bonneau, 2003).

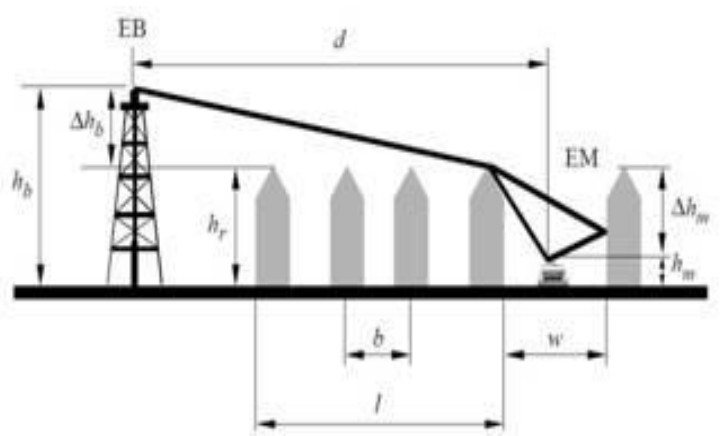

Fig. 1. Parámetros del modelo Walfisch.

Las pérdidas por trayectoria en el modelo Walfisch Ikegami vienen dadas por la ecuación (3) para situaciones sin línea de vista (NLOS) y por la ecuación (4) para situaciones con línea de vista (LOS):

$$
P L(d B)=L_{o}+L_{r t s}+L_{m s d} \quad \text { Para } \quad N L O S
$$

$$
P L(d B)=L_{o} \quad \text { Para LOS }
$$

Donde $L_{o}$ son las pérdidas en el espacio libre y vienen dadas por la ecuación (5):

$$
L_{o}=32,4+20 \log _{10} d+20 \log _{10} f
$$

En la cual, $d$ es la distancia (en Kilómetros) entre la estación base y la estación móvil y $f$ es la frecuencia del enlace en $\mathrm{MHz}$. $L_{r t s}$ corresponde a las pérdidas por difracción azotea-calle y a las pérdidas por dispersión y viene dada por la ecuación (6):

$$
\begin{aligned}
& L_{r t s}=-16,9-10 \log _{10} w+10 \log _{10} f \\
& +20 \log _{10}\left(\Delta h_{\text {mobile }}\right)+L_{\text {ori }}
\end{aligned}
$$

Donde $W$ es el ancho de las calles (en metros), $\Delta h_{\text {mobile }}$ es la diferencia entre la altura media de las edificaciones que rodean a la estación base y la altura de la antena de la estación móvil, y $L_{o r i}$ es una atenuación empírica que depende del ángulo incidente. Finalmente, $L_{m s d}$ son las pérdidas ocasionadas por la difracción azotea-calle y viene dada por la ecuación (7):

$$
\begin{aligned}
& L_{m s d}=L_{b s h}+K_{a}+K_{d} \log _{10} d \\
& +K_{f} 10 \log _{10} f-9 \log _{10} b
\end{aligned}
$$

Donde $L_{b s h}$ y $K_{a}$ representan el aumento de las pérdidas debido a la menor altura de la estación base y $b$ es la distancia entre edificios.

Entre las principales ventajas está el hecho de que puede ser aplicado en escenarios donde la antena del transmisor se encuentra por debajo de la altura media de las edificaciones, a diferencia de lo que ocurre con el modelo Walfisch - Bertoni (Quintana, 2012). Además, este modelo considera la influencia que el ancho de las vías, ángulo de incidencia, altura y separación de edificaciones (entre otros) tiene sobre las pérdidas por trayectoria (Hassan y Mohd, 2013), y así mismo una de sus principales desventajas tiene que ver con la complejidad que en ocasiones se presenta al momento de obtener dicha información que en 
ocasiones debe ser ignorada como en (Hamin y Jamlos, 2014), por lo cual se recurre a los valores recomendados en (Directorate-General Information society: Telecommunications, Markets, 1999) y (ITU-R, 2013) lo cual puede ocasionar una menor aproximación a las pérdidas. Además, su confiabilidad disminuye si el terreno no es plano. Este modelo es recomendado por el sector de radiocomunicaciones de la Unión Internacional de Telecomunicaciones (UIT-R) para su uso en las actividades normativas de las comunicaciones móviles internacionales (IMT-2000).

\subsection{Modelo Interino de la Universidad de Stanford} (SUI)

El modelo Interino de la Universidad de Stanford o SUI (Stanford University Interim) es una extensión del modelo HATA y fue planteado en 1999. Se deriva de mediciones realizadas en 95 macroceldas en diferentes puntos de los Estados Unidos. Es válido para altura de la antena de la estación base entre 10 y 80 metros, altura de la antena de la estación de la estación móvil entre 2 y 3 metros y para un rango de frecuencia entre 0 y $2000 \mathrm{MHz}$ (Erceg et al., 1999). El modelo SUI distingue tres tipos de terrenos (Ardila, 2012):

TIPO A: Relacionado a un escenario con la mayor cantidad de pérdidas, adecuado para terreno montañoso o con colinas, y con moderada o abundante presencia de vegetación.

TIPO B: Asociado a pérdidas intermedias, puede tratarse de terreno plano con abundantes árboles o terreno montañoso con poca vegetación.

TIPO C: Asociado con la menor cantidad de pérdidas, adecuado para escenarios planos con poca presencia de vegetación.

Las pérdidas para el modelo SUI vienen dadas por la ecuación (8):

$$
P L(d B)=A+10 \gamma \log _{10} \frac{d}{d_{o}}+X_{f}+X_{h}+S
$$

Donde $A$ es una cantidad que viene dada por las pérdidas por trayectoria en el espacio libre mostrada en la ecuación (9):

$$
A=20 \log _{10}\left(\frac{4 \pi d_{o}}{\lambda}\right)
$$

Donde $d_{o}$ es una distancia de referencia igual a 100 metros y $\lambda$ es la longitud de onda. $Y$ corresponde al exponente de pérdidas por dispersión dado en la ecuación (10):

$$
\gamma=a-b h_{b}+c / h_{b}
$$

En la cual, $h_{b}$ es la altura de la estación base, $a, b$ y c son contantes. $X_{f}$ y $\mathrm{X}_{h}$ son factores de corrección para la frecuencia y para la altura del receptor respectivamente y mostrados en las ecuaciones 11 y 12 :

$$
\begin{aligned}
& X_{f}=6 \log _{10}\left(\frac{f}{2000}\right) \\
& X_{h}=H \log _{10}\left(\frac{h_{r}}{2000}\right)
\end{aligned}
$$

Por último, $S$ es el factor de desvanecimiento y al igual que otros términos del modelo, su valor se encuentra en la tabla 1.

\section{Tabla 1: Parámetros del modelo SUI}

\begin{tabular}{cccc}
\hline \multirow{2}{*}{$\begin{array}{c}\text { Parámetros del } \\
\text { modelo }\end{array}$} & \multicolumn{3}{c}{ Categoría del terreno } \\
& Terreno & Terreno & Terreno \\
& $\mathbf{A}$ & $\mathbf{B}$ & C \\
\hline A & 4,6 & 0,0075 & 12,6 \\
B & 4 & 0,0065 & 17,1 \\
C & 3,6 & 0,005 & 20 \\
S & 10,6 & 9,6 & 8,2 \\
H & 10,8 & 10,8 & 20 \\
\hline
\end{tabular}

La principal novedad y ventaja de este modelo es que el exponente de pérdidas por trayectos y la desviación estándar del desvanecimiento varían aleatoriamente al salir de una celda y entrar a otra, y esto se modela estadísticamente por medio de variables gaussianas aleatorias cuyos parámetros dependen del tipo de terreno y de la altura del móvil (Erceg et al., 1999). Además este modelo se desvía un poco de los modelos tradicionales al no considerar escenarios urbanos o suburbanos y enfocarse en los tipos de terreno. Se presenta una aproximación menor de las pérdidas para celdas con tamaños mayores a 10 kilómetros.

\section{RECOLECCIÓN DE DATOS}

El área geográfica seleccionada para el estudio presenta en su mayoría características propias de lo que se ha clasificado, desde el punto de vista de propagación radioeléctrica, como ambiente suburbano de acuerdo a la clasificación de entornos 
definidos en (ITU-R, 2013). Con el fin de comparar modelos de pérdidas por trayecto, se tomaron los datos de un conjunto de mediciones de potencia obtenidas a partir de un sistema de drive test. Las pérdidas por trayectoria fueron calculadas para catorce estaciones del área suburbana de la ciudad de Barranquilla, Colombia. La ubicación de cada una de las estaciones base y la ruta seguida durante las mediciones se muestra en la figura 2.

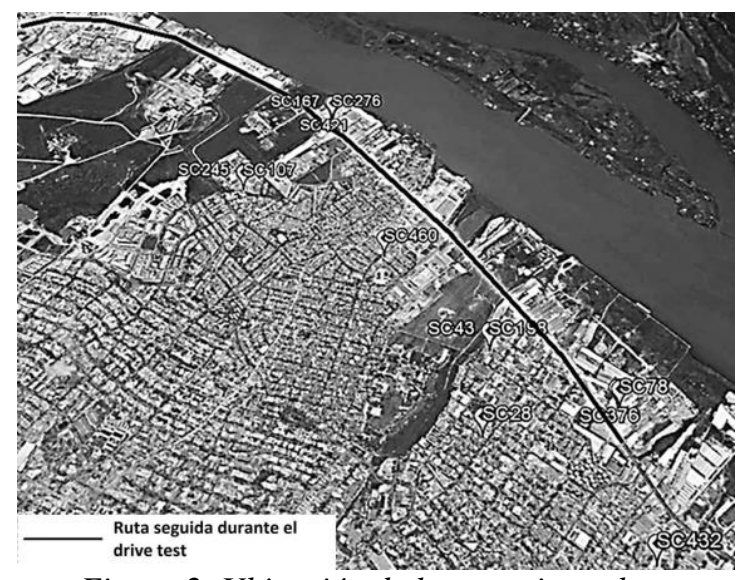

Figura 2. Ubicación de las estaciones base y ruta seguida para el estudio.

La potencia recibida $\left(P_{r x}\right)$ en la estación móvil para cada punto de medición ubicado a una distancia $(d)$ del transmisor está dada por la ecuación (13), de la cual se despeja $P L$ y se obtienen las pérdidas por trayecto (Phillips et al., 2013):

$$
P_{r x}=P_{t x}+G_{t x}+G_{r x}-P L
$$

Donde $P_{r x}$ es la potencia medida en la estación móvil, $P_{t x}$ es la potencia de transmisión de la estación base, $G_{t x}$ es la ganancia de la antena del transmisor y $G_{r x}$ es la ganancia de la antena del receptor.

La configuración de los parámetros para la medición fue realizada de acuerdo a las recomendaciones del Reporte Final COST-231 y de la UIT (Directorate-General Information society: Telecommunications, Markets, 1999) (ITU-R, 2013). Para la recolección de los datos se hizo el recorrido de la ruta (a una velocidad promedio de $40 \mathrm{Km} / \mathrm{h}$ ) en un vehículo equipado con la herramienta TEMS Versión 14.2 que permite medir la potencia de recepción y además muestra la estación base de la cual el dispositivo móvil obtiene la mejor recepción. El computador, con el TEMS incorporado, se conecta a un dispositivo de posicionamiento global (GPS) y a un dispositivo móvil que recibe la señal del operador. La ganancia de la antena en el receptor era inferior a $1 \mathrm{dBm}$. El GPS determina las coordenadas geográficas de cada punto donde se realiza una medición y en el computador se almacena toda la información recolectada. La banda de frecuencia en la que se realizaron las mediciones fue la de los $1900 \mathrm{MHz}$ y la información de las estaciones base que intervinieron en el estudio fue proporcionada por el operador. En total se tomaron 41551 mediciones de potencia en el equipo receptor.

Para determinar la efectividad de los modelos de propagación se ha realizado una comparación entre la pérdida por trayectoria pronosticada para cada modelo y la pérdida por trayecto calculada a partir de las mediciones de potencia en una de las estaciones base del ambiente sub-urbano. Las ecuaciones (14) y (15) son utilizadas para calcular el error de predicción y el error de predicción en términos de porcentaje respectivamente (Bhuvaneshwari et al., 2013):

$$
\begin{aligned}
& \delta_{\text {error }}=P L_{\text {medida }}-P L_{\text {modelo }} \\
& \delta_{\text {error }}=\left[\left(P L_{\text {medida }}-P L_{\text {modelo }}\right) / P L_{\text {medida }}\right] * 100 \%
\end{aligned}
$$

Donde $P L_{\text {medida }}$ es la pérdida obtenida a partir de las mediciones de potencia de potencia, $P L_{\text {modelo }}$ es la pérdida obtenida con cada modelo y $\delta_{\text {error }}$ es el error de predicción.

\section{RESULTADOS}

La comparación se hizo con los cálculos obtenidos de los modelos COST 231-HATA, WalfischIkegami y SUI (Tipo B) para mediciones tomadas en el área de cobertura de la celda situada en las coordenadas $11^{\circ} 1^{\prime} 49^{\prime}, \mathrm{N}$ de latitud y 78 48'25.04' 'O de longitud. La antena de transmisión (propiedad de un operador local), es el modelo K742215 fabricada por Kathrein y hace parte de la celda identificada por el operador como SC276. Esta antena tiene doble polarización $\left( \pm 45^{\circ}\right)$, con ganancia de $18 \mathrm{dBi}$ y está ubicada a 30 metros radiando a una potencia de 20 Watts en la banda de los $1900 \mathrm{MHz}$. Los valores calculados de las pérdidas por trayectoria (a partir de las mediciones de potencia en el escenario estudiado) y las pérdidas por trayectoria calculadas correspondientes al área sub urbana de Barranquilla, se trazan en función de la distancia y se muestran en la figura 4 y 5 respectivamente. 


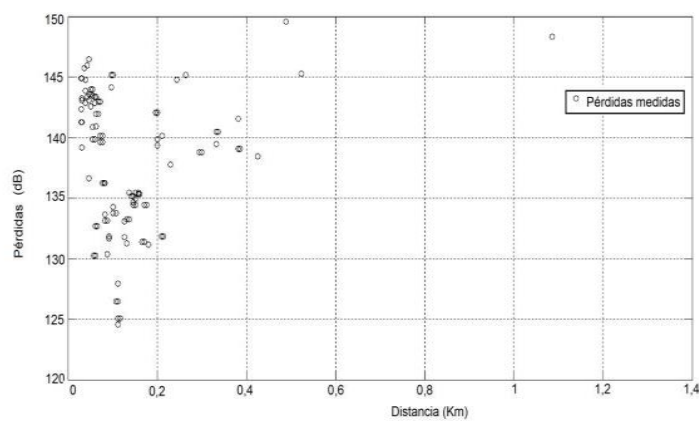

Figura 4. Pérdidas medidas Vs distancia en el área de cobertura de la Estación Base SC276

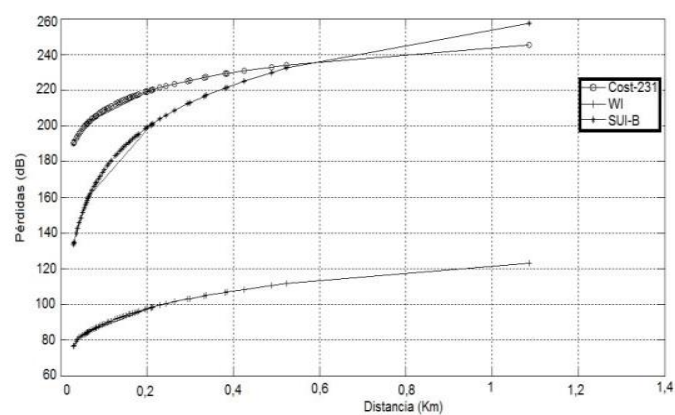

Figura 5. Pérdidas calculadas con cada modelo Vs distancia en el área de cobertura de la Estación Base SC276

Para la evaluación del desempeño de los modelos de propagación, se calculó la media de las pérdidas por trayectoria, el error de predicción y el error relativo de cada modelo con respecto a las mediciones. Errores con medias que tienden a cero indican que los modelos describirían correctamente las pérdidas por trayecto en los escenarios, y a medida que la media del error aumenta se presenta sobreestimación por parte de los modelos. El análisis de los errores comparativos para cada modelo en el escenario sub urbano es mostrado en las tablas 2, 3 y 4 .

Tabla 2: Análisis del error para el modelo COST 231-HATA

\begin{tabular}{ccccc}
\hline $\begin{array}{c}\text { Estación } \\
\text { Base }\end{array}$ & $\begin{array}{c}\text { Pérdidas } \\
\text { Medidas }\end{array}$ & $\begin{array}{c}\text { Perdida por } \\
\text { trayectoria } \\
\text { promedio } \\
(\mathrm{dB})\end{array}$ & $\begin{array}{c}\text { Error de } \\
\text { Predicción } \\
(\mathrm{dB})\end{array}$ & $\begin{array}{c}\text { Error } \\
\text { relativo } \\
(\%)\end{array}$ \\
\hline SC276 & 138,1 & 209,5 & $-71,4$ & 52,1 \\
\hline
\end{tabular}

Tabla 3: Análisis del error para el modelo Walfisch-Ikegami

\begin{tabular}{ccccc}
\hline $\begin{array}{c}\text { Estación } \\
\text { Base }\end{array}$ & $\begin{array}{c}\text { Pérdidas } \\
\text { Medidas }\end{array}$ & $\begin{array}{c}\text { Perdida por } \\
\text { trayectoria } \\
\text { promedio } \\
(\mathrm{dB})\end{array}$ & $\begin{array}{c}\text { Error de } \\
\text { Predicción } \\
(\mathrm{dB})\end{array}$ & $\begin{array}{c}\text { Error } \\
\text { relativo } \\
(\%)\end{array}$ \\
\hline SC276 & 138,1 & 90,6 & 47,5 & 34,7 \\
\hline
\end{tabular}

Tabla 4: Análisis del error para el modelo Interino de la Universidad de Stanford Tipo B

\begin{tabular}{ccccc}
\hline $\begin{array}{c}\text { Estación } \\
\text { Base }\end{array}$ & $\begin{array}{c}\text { Pérdidas } \\
\text { Medidas }\end{array}$ & $\begin{array}{c}\text { Perdida por } \\
\text { trayectoria } \\
\text { promedio } \\
(\mathrm{dB})\end{array}$ & $\begin{array}{c}\text { Error de } \\
\text { Predicción } \\
(\mathrm{dB})\end{array}$ & $\begin{array}{c}\text { Error } \\
\text { relativo } \\
(\%)\end{array}$ \\
\hline SC276 & 138,1 & 176,8 & $-38,7$ & 29,3 \\
\hline
\end{tabular}

Valores altos de errores de predicción y error relativo indican que el modelo se aleja de las estimaciones de las pérdidas por trayectoria en el ambiente estudiado. Los cálculos muestran que el error relativo promedio del modelo SUI Tipo B es de $29,3 \%$ en el ambiente sub-urbano, siendo más bajo que en el caso de los modelos COST-231 HATA y Walfisch Ikegami para ese mismo escenario; lo cual indica un bajo rendimiento en el nivel de predicción de las pérdidas por trayectoria por parte de estos modelos. Con el modelo SUI Tipo B, el 75,87\% de las mediciones tiene un error entre $-22,5$ y $18,75 \mathrm{~dB}, \quad \mathrm{y}$ el $9,6 \%$ de las mediciones arrojan valores de error de predicción entre $-7,5 \mathrm{~dB}$ y 7,5 dB.

A pesar que la mayoría de los valores de altura de las antenas, frecuencia del enlace, distancia de cobertura y tipo de celda están dentro del rango de validez para los modelos COST-231 HATA y Walfisch-Ikegami, las tablas 3 y 4 muestran errores de predicción que no son típicos dentro de la literatura para estudios similares. Sin embargo puede encontrarse en la revisión algunos ejemplos de investigaciones similares donde el error está por encima del orden de los $15 \mathrm{~dB}$ que típicamente se obtienen para el modelo COST-231 HATA como ocurre en (Bhuvaneshwari y Sathyasavithri, 2013), o donde el error medio cuadrático resulta ser el doble de lo obtenido con el modelo SUI (Chebil et al., 2011).

La figura 4 muestra que la mayoría de las mediciones se realizaron a una distancia entre 0 y 200 metros entra la antena de transmisión y de recepción, lo cual reduce los niveles de predicción del modelo COST 231-HATA. En comparación, el modelo Walfisch-Ikegami presenta una mejor aproximación a las mediciones cuando la distancia 
entre la estación base y el móvil es menor a un kilómetro de distancia.

También se observa que en el escenario estudiado predominan las edificaciones de dos o tres pisos, pero también se presentan algunas construcciones de cinco o más pisos, las cuales no corresponden a la clasificación de ambiente suburbano presentado por la UIT-R en (ITU-R, 2013). Otra característica del área seleccionada es que muchas edificaciones se encuentran separadas por varios metros de las estructuras a su alrededor y además existen algunas zonas no pobladas y sin edificaciones a lo largo de la ruta del drive test, tal como puede observarse en la figura 2. Lo anterior implica que en la mayoría del recorrido no se mantiene una configuración urbanística típica de los escenarios originales en los que originalmente se desarrolló el modelo Walfisch-Ikegami, lo cual afecta sus niveles de aproximación.

\section{CONCLUSIONES}

Tres modelos empíricos de pérdidas por trayectoria fueron analizados en este artículo: COST-231 HATA, Walfisch Ikegami e Interino de la Universidad de Stanford; los cuales fueron comparados con las pérdidas por trayectoria obtenidas a partir de mediciones de potencia realizadas en un escenario suburbano de la ciudad de Barranquilla durante un drive test.

Los resultados muestran una sobrestimación de las pérdidas de cada uno de los modelos estudiados en el ambiente suburbano estudiado, Sin embargo en los primeros 200 metros el modelo Interino de la Universidad de Stanford tiene una mayor aproximación a las mediciones. El modelo SUI tipo B presenta un error relativo más bajo en comparación con los otros estudiados, sin embargo sus altos valores de error y el hecho de que menos del $10 \%$ de las mediciones se encuentran en el rango de diferencia de $7,5 \mathrm{~dB}$ indican que se hace necesario realizar el ajuste del modelo con el propósito de establecer si es posible aumentar los niveles de eficiencia en la predicción. La presencia, en la zona seleccionada para el estudio, de algunas áreas sin edificaciones y sin vegetación indica que se debería considerar la evaluación del modelo SUI tipo C, el cual está asociado a escenarios con pocas pérdidas.

Los resultados también muestran que el modelo que presenta la aproximación más baja en relación con las mediciones es el modelo COST-231 HATA, el cual presenta valores de error relativo más alto, llegando a ser del orden del 52,1\%. Se observan valores de error de predicción muy por encima de los $15 \mathrm{~dB}$ típicamente registrados en la literatura, por lo cual se hace necesario un cuidadoso ajuste que permita aumentar la aproximación a las pérdidas.

En lo que respecta al modelo Walfisch-Ikegami, el terreno plano y la disposición de las calles de la zona seleccionada favorece las condiciones de este modelo. Sin embargo, los niveles de predicción se ven afectados por la disposición irregular de las edificaciones que rodean a la estación base, ya que no es propia de las zonas donde originalmente se desarrolló este modelo. Se aconseja el uso de herramientas cartográficas computacionales que permitan obtener datos más cercanos a la realidad en lo que respecta a las estructuras que rodean a las estaciones base usadas en las mediciones; con el fin de tener mayor información acerca de la verdadera estimación entregada por el Modelo Walfisch Ikegami.

Como trabajo futuro se recomienda ampliar la campaña de medición a todo el escenario suburbano de la ciudad de Barranquilla para verificar la validez de estos modelos en los diferentes entornos urbanísticos de la ciudad y así obtener un algoritmo de predicción de pérdidas por trayectoria que posibilite el ajuste de forma automática teniendo en cuenta las condiciones específicas de cada zona.

\section{REFERENCIAS}

Acar T. (2015). Comparison of computer-based propagations models with experimental data collected in an urban area at $1800 \mathrm{MHz}$. 2015 IEEE 16th Annual Wireless and Microwave Technology Conference.

Ardila C. (2012). Ajuste estadístico de modelos de propagación de señal usando medidas de la ciudad de Cali. Ingenium 6, 1-8.

Bhuvaneshwari A., y Sathyasavithri, T. (2013). Comparative analysis of mobile radio path loss models for suburban environment in Southern India. 2013 International Conference on Emerging Trends in VLSI, Embedded System, Nano Electronics and Telecommunication System.

Bhuvaneshwari A., Hemalatha, R., y Satyasavithri, T. (2013). Development of an Empirical Power model and Path Loss Investigations 
for Dense Urban Region in Southern India. 2013 IEEE 11th Malaysia International Conference on Communications.

Chebil J., Lwas A. K., Islam M. R., y Zyoud, A.H. (2011). Comparison of empirical propagation path loss models for mobile communications in the suburban area of Kuala Lumpur. 2011 4th International Conference on Mechatronics.

Directorate-General Information society: Telecommunications, Markets, T.I. and E. of R. (1999). COST Action 231 - Final report.

Erceg V., Member, S., Greenstein, L. J., Tjandra, S. Y., Parkoff, S. R., Gupta, A., y Bianchi, R. (1999). An Empirically Based Path Loss Model for Wireless Channels in Suburban Environments. Ieee Journal on Selected Areas in Communications, 17.

Garcia J. C., y Rogriguez, O. A. (2002). Desempeño de Modelos de Propagación en Comunicación Móvil para la zona de Caldas Parte 1: Modelos para áreas urbanas. X Congreso Internacional de Telecomunicaciones.

Hamin S. F., y Jamlos M. F. (2014). An overview of outdoor propagation prediction models. 2014 IEEE 2nd International Symposium on Telecommunications Technologies.

Herrera J. E. (2013). Predicción de cobertura en ambientes semiurbanos mediante método experimental para sistemas inalámbricos en la banda de frecuencia de $2.4 \mathrm{GHz}$. Revista Colombiana de Tecnologías de Avanzada, Vol 2, N 22 pp. 68-77.

Herrera J. E., y Hernández C. A. (2016). Análisis experimental de cobertura en una red mallada predecible aplicando modelos de regresión no lineal en interiores. Revista Colombiana de Tecnologías de Avanzada, Vol 2, N 28 pp. 105-114.

Herrera J. E., y Pérez N. A. (2008). Modelo de propagación en ambientes exteriores para sistemas inalámbricos operando en la banda de frecuencias de $2.4 \mathrm{GHz}$. Revista Colombiana de Tecnologías de Avanzada, Vol 2, N 12 pp. 114-124.

ITU-R. (2013). Datos de propagación y métodos de predicción para la planificación de los sistemas de radiocomunicaciones de exteriores de corto alcance y redes de radiocomunicaciones de área local en la gama de frecuencias de $300 \mathrm{MHz}$ a $100 \mathrm{GHz}$. http://www.itu.int/dms_pubrec/itu-r/rec/p/RREC-P.1411-7-201309-I!!PDF-S.pdf

(Consultado: julio 20 de 2015)

Phillips C., Sicker, D., y Grunwald, D. (2013). A Survey of Wireless Path Loss Prediction and Coverage Mapping Methods. IEEE Communications Surveys \& Tutorials.

Quintana R. A. (2012). Evaluación de modelos de propagación de canal inalámbrico. Revista Cubana de Ingeniería.

Rappaport T. S. (2002). Wireless Communications: Principles and Practice (Segunda ed.). Editorial Prentice Hall.

Hassan R., y Mohd F. (2013). Comparative Study on Radio Wave Propagation Models for $4 G$ Network. 2013 15th International Conference on Advanced Communications Technology.

Salazar M., y Bonneau, R. J. (2003). A survey of various propagation models for mobile communication. Antennas and Propagation Magazine, 45, 239-307. Retrieved from Base de datos de IEEE

Tahat A., y Alqudah, Y. (2011). Analysis of propagation models at $2.1 \mathrm{GHz}$ for simulation of a live $3 G$ cellular network. 2011 Wireless Advanced. 